\title{
Contextual cuing by global features
}

\author{
MELINA A. KUNAR \\ Harvard Medical School, Boston, Massachusetts \\ and Brigham and Women's Hospital, Boston, Massachusetts \\ STEPHEN J. FLUSBERG \\ Brigham and Women's Hospital, Boston, Massachusetts \\ and \\ JEREMY M. WOLFE \\ Harvard Medical School, Boston, Massachusetts \\ and Brigham and Women's Hospital, Boston, Massachusetts
}

\begin{abstract}
In visual search tasks, attention can be guided to a target item-appearing amidst distractors-on the basis of simple features (e.g., finding the red letter among green). Chun and Jiang's (1998) contextual cuing effect shows that reaction times (RTs) are also speeded if the spatial configuration of items in a scene is repeated over time. In the present studies, we ask whether global properties of the scene can speed search (e.g., if the display is mostly red, then the target is at location X). In Experiment 1A, the overall background color of the display predicted the target location, and the predictive color could appear 0,400, or 800 msec in advance of the search array. Mean RTs were faster in predictive than in nonpredictive conditions. However, there was little improvement in search slopes. The global color cue did not improve search efficiency. Experiments $1 \mathrm{~B}-1 \mathrm{~F}$ replicated this effect using different predictive properties (e.g., background orientation-texture and stimulus color). The results showed a strong RT effect of predictive background, but (at best) only a weak improvement in search efficiency. A strong improvement in efficiency was found, however, when the informative background was presented $1,500 \mathrm{msec}$ prior to the onset of the search stimuli and when observers were given explicit instructions to use the cue (Experiment 2).
\end{abstract}

To interact with the world, we often have to parse visual information into relevant and irrelevant stimuli. For example, when searching for a friend in a crowd, we must ignore non-goal-relevant information (such as other people's faces or inanimate objects) in order to select the correct face. If we were to pay attention to every visual stimulus, we might become overwhelmed and thus could not perform many important tasks.

In the laboratory, we can study how we process visual information by asking observers to search for a target item among a number of distracting items and record the reaction time (RT) taken to respond. We can use these measurements to understand how we process the world. For example, if we measure RT as a function of set size (the number of items in the display), then the slope of the RT $\times$ set size function gives an estimate of the cost of adding items to the display and is a measure of search efficiency. If attention can be deployed to a target independently of the number of distractor items, then the slope will be $0 \mathrm{msec} / \mathrm{item}$, and the visual search task can be la-

This research was supported by a grant from the National Institute of Mental Health to J.M.W. The authors thank Diana Ye for her help with the initial data collection, as well as Marvin Chun and two anonymous reviewers for their helpful comments. Address correspondence to M. A. Kunar, Visual Attention Laboratory, 64 Sidney Street, Suite 170, Cambridge, MA 02139 (e-mail: kunar@search.bwh.harvard.edu). beled "efficient." According to a Guided Search model of attention (see, e.g., Wolfe, 1994; Wolfe, Cave, \& Franzel, 1989), a limited set of features are able to guide attention toward a target (see Wolfe \& Horowitz, 2004, for a review). For example, color-finding the red bar among green bars and orientation-finding a vertical bar among horizontal bars both produce efficient search (Treisman $\&$ Gelade, 1980). The efficiency of search is related to the strength of guidance (Wolfe, 1994; Wolfe et al., 1989). Thus, if participants search for a target defined by a conjunction of properties (e.g., find the red horizontal bar among green horizontals and red verticals), search is less efficiently guided than in a simple feature search and typically produces search slopes of around 5-15 msec/item on target present trials (see, e.g., Treisman \& Sato, 1990; Wolfe, 1998). In the absence of guiding feature information, search is still less efficient, producing target present slopes of around $20-40 \mathrm{msec} / \mathrm{item}$ even when the items are large enough to identify without fixation (e.g., Braun \& Sagi, 1990; Egeth \& Dagenbach, 1991; Wolfe, 1998).

Beyond attributes of the target and distractor items, there are global visual properties that can help us process the world. Participants find it more difficult to search for an object in a jumbled, incoherent scene than for an object in a coherent one (Biederman, 1972; Biederman, Glass, \& Stacey, 1973). Scene information does not need to be meaningful or explicitly recognized. Chun and Jiang 
(1998) have found that participants are faster to respond to a target if it is always accompanied by the same spatial context than if it is not. If participants need to determine the orientation of a T, they are faster to respond when a specific target location is always accompanied by Ls in a unique layout. The benefit of having the target appear in a predictive, repeated display is known as contextual cuing (Chun \& Jiang, 1998; see also Chun, 2000, for a review), and it can be based on implicit knowledge. At the end of the experiment, when performing a two-alternative forcedchoice recognition task, participants were at chance when asked if they had seen a display before (Chun \& Jiang, 1998; see also Chun \& Jiang, 2003).

Given that the spatial context of a scene can produce an RT advantage (or a "cuing" effect), we asked whether other aspects of a scene could produce similar results. In particular, we investigated whether a nonspatial property-like the overall color of a scene-could produce a similar effect in the absence of a consistent spatial layout. We know that features like color are often used to guide our attention to the target item in the face of competing stimuli when those features differentiate the target and distractors. For example, if one is searching for a red item among green distractors, one can efficiently guide attention toward red and away from green (Carter, 1982; Green \& Anderson, 1956; Wolfe, 1994; Wolfe et al., 1989). Can feature information also be useful if it indirectly identifies the target location? If a red display means that the target is in one location and a green display means that it is in another unique location, can the searcher use that information to speed response and, in turn, increase search efficiency?

Please note that although we have mentioned search performance in terms of Guided Search (Wolfe, 1994; Wolfe et al., 1989), any decrease in search efficiency could also be accounted for by other models, such as limited-capacity (see, e.g., Posner, 1980) or unlimited-capacity (e.g., Palmer, 1994; Palmer, Ames, \& Lindsey, 1993; Palmer, Verghese, \& Pavel, 2000; Shaw \& Shaw, 1977; Townsend \& Ashby, 1983) models of attention. Guided Search and other limited attention-capacity models may assume an improvement in search efficiency, since one could orient attention to items appearing around the cued location rather than across all items in the visual field (in fact, Song \& Jiang, 2005, suggest that participants need only encode the configuration of three to four distractor items to find a valid contextual cuing effect). Alternatively, unlimited attention-capacity models (especially those based on signal detection theories; see, e.g., Palmer, 1994, and Palmer et al., 1993) might claim that contextual cuing allows participants to better ignore distractor items. In the present experiments, we did not seek to pitch one attention model over another, but instead to pose the overall question: Does contextual cuing by global features improve search efficiency? For the sake of simplicity, we will refer to such an idea as an improvement in guidance.

In Experiment 1, we investigated whether the average features of a background could speed response and/or aid search efficiency when the background appeared 0, 400, or $800 \mathrm{msec}$ prior to the search stimuli. The results revealed a speeding of mean RT, but no decrease in RT $\times$ set size slopes (thus, no evidence of guidance). Experiments $1 \mathrm{~B}-1 \mathrm{~F}$ were replications of this effect using different predictive properties, such as the background texture or the color of the search stimuli. In Experiment 2, we presented the background $1,500 \mathrm{msec}$ prior to the search stimuli and gave participants the explicit knowledge that some background features were predictive of the target location. In this case, we observed both a speeding of mean RT and a decrease in slope. Global color information can guide search if it is presented explicitly and well in advance of the search display. However, with less lead time and explicit awareness, global predictive properties only produce an RT cuing effect.

\section{EXPERIMENT 1A}

In Experiment 1A, we investigated whether the average background color of a display could speed response if that color predicted the target location.

\section{Method}

Participants. Twelve participants from 18 to 55 years old served as participants. Each participant passed the Ishihara test for color blindness and had normal or corrected-to-normal vision. All participants gave informed consent and were paid for their time.

Apparatus and Stimuli. The experiment was conducted on a Macintosh computer using MATLAB software with the Psychophyics Toolbox (Brainard, 1997; Pelli, 1997). Search displays are shown in Figure 1. The distractor items were $\mathrm{L}$ shapes presented randomly in one of four orientations $\left(0^{\circ}, 90^{\circ}, 180^{\circ}\right.$, or $\left.270^{\circ}\right)$. The target item was a T shape rotated $90^{\circ}$ either to the left or to the right with equal probability. A blue dot at the center of the screen served as a fixation point. Three black concentric circles surrounded the fixation point with diameters of $9.5^{\circ}, 15.5^{\circ}$, and $25^{\circ}$. Sixteen black lines radiated out from the fixation point roughly equidistant from one another to form a spider web lattice. On every trial, either 8 or 12 (depending on the set size) circular "placeholders" appeared at locations where a spoke crossed a concentric circle. Because visual acuity declines with distance from the fixation point, the size of the place-holding circles and of the search stimuli increased with eccentricity. Those on the closest concentric circle were $2^{\circ}$ in diameter, those on the middle concentric circle were $3.3^{\circ}$, and those on the farthest concentric circle were $5.4^{\circ}$. The background measured $29^{\circ} \times 29^{\circ}$ and was composed of a texture of different-sized, overlapping circles that were filled with variations of a single color. There were eight background colors: yellow, red, dark blue, orange, cyan, green, purple, or gray. The background color changed from trial to trial. Each color was equally probable. Please note that, within a background, the global texture remained unchanged (e.g., the background was overall red); however, on each successive display, the local properties varied (e.g., the color and size of the individual circles making up the display changed across each red display).

All stimuli were made up of two lines of equal length (forming either an L or a T). Ts and Ls appeared within the circular placeholders. Stimuli enclosed in the smallest placeholders subtended a visual angle of $1^{\circ} \times 1^{\circ}$, those enclosed in the middle placeholders subtended $1.5^{\circ} \times 1.5^{\circ}$, and those enclosed in the largest placeholders subtended $2.5^{\circ} \times 2.5^{\circ}$. The targets, distractors, and outline of the placeholders were of the same color as the overall background, whereas the inside of the placeholders was dark gray.

Procedure. A tone sounded at the start of each trial. There were 10 practice trials, followed by 512 experimental trials divided-for 


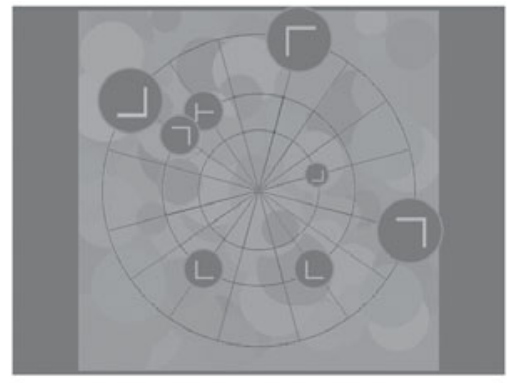

SOA $0 \mathrm{msec}$

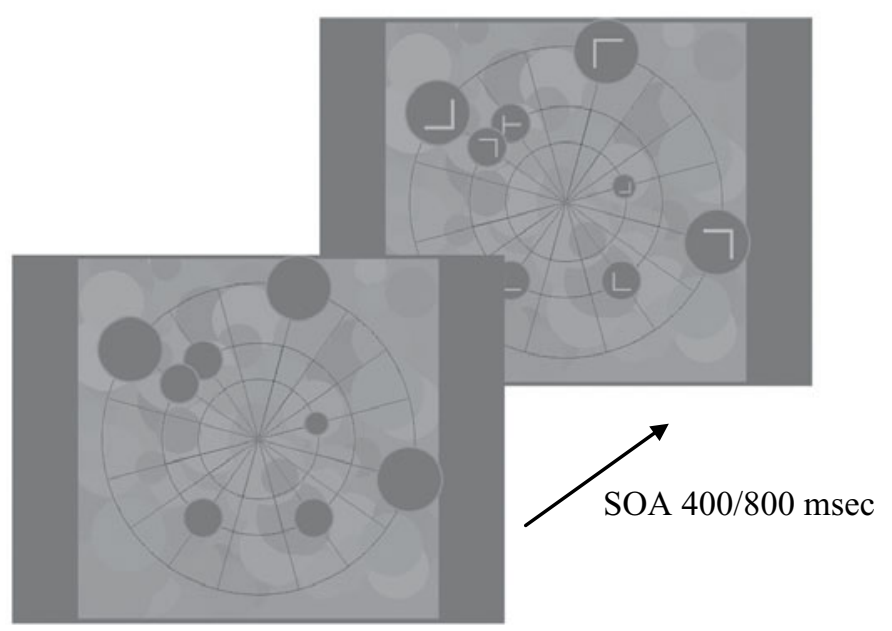

Figure 1. Example displays for Experiment 1A.

analytic purposes - into 8 epochs of 64 trials each. Approximately half of the trials in each epoch had a set size of 8 ; the remaining trials had a set size of 12 .

Each participant was tested in three conditions: (1) a search task in which the background appeared simultaneously with the search stimuli, (2) a search task in which the appearance of the background preceded that of the stimuli by $400 \mathrm{msec}$, and (3) a search task in which the appearance of the background preceded that of the stimuli by $800 \mathrm{msec}$. In each condition, participants were asked to respond to whether the bottom of the target letter, T, was facing left (by pressing the letter "a" on the keyboard) or whether it was facing right (by pressing the letter "l"). For Epochs 1 to 7, four of the background colors were always associated with a specific target location (e.g., if the display was purple, then the target was always at location X, regardless of set size or spatial layout). The other four colors did not predict the target location (i.e., the target changed its location on every presentation of the color). The four predictive colors were randomly assigned within each condition. In Epoch 8, the target locations for both the predictive and random trials changed with every presentation of the colored background (i.e., the predictive colors no longer predicted the target location). If participants used the background information in Epochs 1-7, it would no longer be beneficial in Epoch 8, therefore leading us to predict an increase in RTs. Each SOA condition was blocked, and the order of the blocks was randomized. Feedback was given after each trial.

\section{Results and Discussion}

Figure 2 shows the RTs and slopes for each set size in the predictive and nonpredictive (random) conditions.
Different graphs show different SOA conditions. Table 1 shows the low overall error rates. Because they were not indicative of any speed-accuracy trade-off, we will not discuss them further and will instead concentrate on RT and slope analysis. RTs that were below $200 \mathrm{msec}$ and above 4,000 msec were eliminated. This led to the removal of less than $1 \%$ of the data.

For SOA $0 \mathrm{msec}$, the RT data shows that there was an overall reduction in RTs when the background color was predictive rather than random (Epochs 1-7). This effect was reliable for set size $8[F(1,11)=10.5, p<.01]$ and for set size $12[F(1,11)=5.9, p<.05]$. In order to examine how learning influences performance, previous contextual cuing experiments compared predictive and random RTs averaged across the latter part of the experiment (Epochs 5-7, following the design of Chun \& Jiang, 1998 and Jiang, Leung, \& Burks, 2006, who also collapsed RTs across the last three predictive epochs). Using this measurement, we see with set size 8 that there was a 74-msec RT benefit of having a predictive background $[t(11)=-5.4, p<.01]$, and at set size 12 there was a 91msec benefit $[t(11)=-2.7, p<.05]$. The reliable difference between predictive and random RTs disappeared at Epoch 8, where all trials were random. These data suggest that RTs become faster as participants learn the association between background and target location, rather than 
Table 1

Error Percentages for Experiments 1A-1F and Experiment 2

\begin{tabular}{|c|c|c|c|c|c|c|c|c|c|}
\hline & \multirow{3}{*}{$\begin{array}{c}\text { SOA } \\
(\mathrm{msec})\end{array}$} & \multicolumn{8}{|c|}{ Condition } \\
\hline & & \multicolumn{4}{|c|}{ Predictive } & \multicolumn{4}{|c|}{ Random } \\
\hline & & 8 & 12 & 18 & 24 & 8 & 12 & 18 & 24 \\
\hline \multirow[t]{3}{*}{ Experiment $1 \mathrm{~A}$} & 0 & 1.3 & 0.4 & & & 0.9 & 1.2 & & \\
\hline & 400 & 1.0 & 0.9 & & & 1.4 & 1.1 & & \\
\hline & 800 & 1.1 & 0.5 & & & 1.0 & 1.1 & & \\
\hline \multirow[t]{3}{*}{ Experiment 1B } & 0 & 1.7 & 1.9 & & & 2.7 & 1.4 & & \\
\hline & 400 & 1.8 & 2.4 & & & 1.8 & 2.0 & & \\
\hline & 800 & 2.7 & 3.0 & & & 2.0 & 2.5 & & \\
\hline \multirow[t]{3}{*}{ Experiment $1 \mathrm{C}$} & 0 & 2.4 & 2.0 & & & 2.6 & 2.7 & & \\
\hline & 400 & 1.6 & 1.8 & & & 1.1 & 1.3 & & \\
\hline & 800 & 1.2 & 1.1 & & & 1.3 & 1.4 & & \\
\hline \multirow[t]{3}{*}{ Experiment 1D } & 0 & 2.5 & 1.7 & & & 2.0 & 2.7 & & \\
\hline & 400 & 2.4 & 2.1 & & & 2.3 & 2.4 & & \\
\hline & 800 & 1.7 & 2.5 & & & 2.3 & 2.6 & & \\
\hline Experiment 1E & 0 & 1.3 & 1.4 & & & 1.4 & 1.1 & & \\
\hline Experiment 1F & 0 & & & 2.5 & 2.3 & & & 2.6 & 3.3 \\
\hline Experiment 2 & 1,500 & 2.7 & 2.7 & & & 1.3 & 1.3 & & \\
\hline
\end{tabular}

the actual target location per se. In Epoch 8, the target locations for the predictive trials remained the same; however, the predictive backgrounds changed. If it had been the absolute target location that was important, RTs for previously predictive trials (i.e., those that had the same target locations) should still have been faster than those for the nonpredictive trials. It may have also been possible that participants learned the backgrounds were no longer predictive in Epoch 8; however, even if this were true, the data still suggest that it was the featural background rather than the absolute target location that was important.

Slopes are shown in the right-hand panels of Figure 2. When we examine the search slope data for SOA $0 \mathrm{msec}$, we see that there was no benefit in search when the background was predictive. Search slopes for Epochs 1-7 were not more efficient when the background was predictive rather than random $[F(1,11)=0.2, p=.68]$. Nor was the average slope-derived from Epochs 5-7-more efficient in the predictive condition $[t(11)=-0.6, p=.58]$.

The results of the SOA $0-\mathrm{msec}$ condition show that there was a clear RT advantage of a predictive background, but that this did not affect search slopes. Perhaps a guidance effect on search slope would have occurred if the background information had been available earlier. However, the data from SOA $400 \mathrm{msec}$ and SOA $800 \mathrm{msec}$ fail to support this hypothesis. If anything, providing background foreknowledge may weaken the chance for this effect to occur. Figures $2 \mathrm{~B}$ and $2 \mathrm{C}$ show the RTs and slopes for the SOA 400-msec and SOA 800-msec conditions, respectively. The RT data for SOA $400 \mathrm{msec}$ show that there was a main effect of the predictability of the background: $[F(1,11)=8.3, p<.05]$ and $[F(1,11)=$ $16.8, p<.01]$ for set sizes 8 and 12 , respectively. Over Epochs 5-7, there was a 58-msec advantage of having a predictive background for set size $8[t(11)=-2.2, p=$ $.05]$ and a 113-msec advantage for set size $12[t(11)=$ $-3.7, p<.01]$. At SOA $800 \mathrm{msec}$, there was not a main effect of the predictability of the background: $[F(1,11)=$
$0.5, p=.51]$ and $[F(1,11)=3.2, p=.10]$ for set sizes 8 and 12, respectively. Over Epochs 5-7, there was only a marginal advantage $(35 \mathrm{msec})$ at set size $8[t(11)=-2.0$, $p=.07]$, and although there was a $36-\mathrm{msec}$ advantage at set size 12 , this is not reliable $[t(11)=-1.5, p=.15]$. The RT data suggest that there was some benefit of having a predictive background, although this was more apparent at SOA $400 \mathrm{msec}$ than at SOA $800 \mathrm{msec}$.

Turning to the search slope data for SOAs 400 and $800 \mathrm{msec}$, we find that there was still no benefit to search efficiency when the background was predictive rather than random. At SOA $400 \mathrm{msec}$, there appears to have been a slight guidance benefit of having a predictive background; however, this was weak and did not replicate (see Experiments 1B-1F for details). Here, although the main effect across epochs was marginally significant $[F(1,11)=4.3$, $p=.06]$, the difference in predictive and random slopes for Epochs $5-7$ was not reliable $[t(11)=-1.6, p=.13]$. Similarly, there was no reliable effect on slope for SOA $800 \mathrm{msec}$ [main effect, $F(1,11)=1.7, p=.22$; Epochs $5-7$, $t(11)=-0, p=.97]$. Notice that even if the slope effect at $400 \mathrm{msec}$ were significant, the slope was only reduced to an inefficient $30 \mathrm{msec} / \mathrm{item}$ - comparable to that seen for Ts among Ls in the absence of any guidance in other experiments (see, e.g., Wolfe, Oliva, Horowitz, Butcher, \& Bompas, 2002).

The results of Experiment 1A suggest that the featural properties of the background can speed the response to the target. However, this facilitation does not appear to be the result of the background information guiding attention toward likely target locations. Guidance should produce a reduction in the effective set size and, thus, a reduction in the slope of the RT $\times$ set size functions. This was not found. Increasing the time between the onset of the predictive/nonpredictive background - and hence the amount of "warning" or preparation time given to a participant - did not produce more efficient guidance. If anything, precuing with the background seemed to weaken the featural cuing effect. 

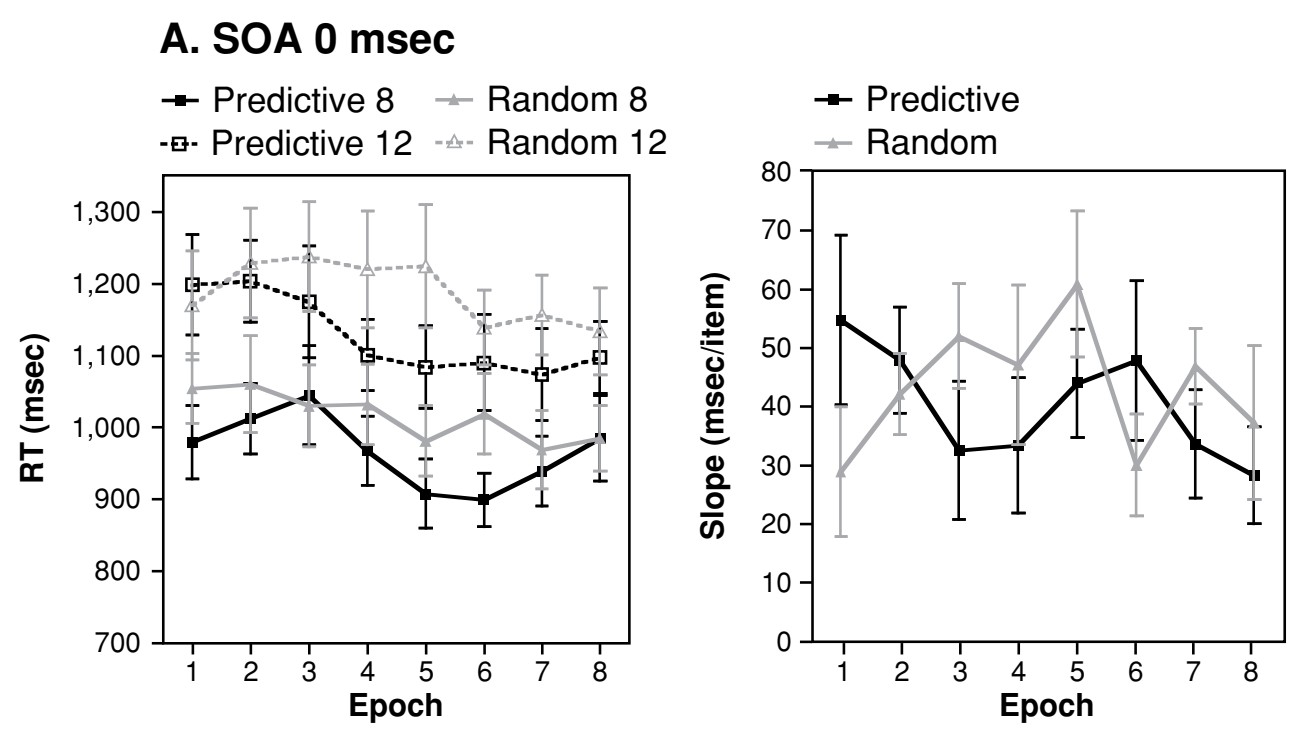

B. SOA $400 \mathrm{msec}$
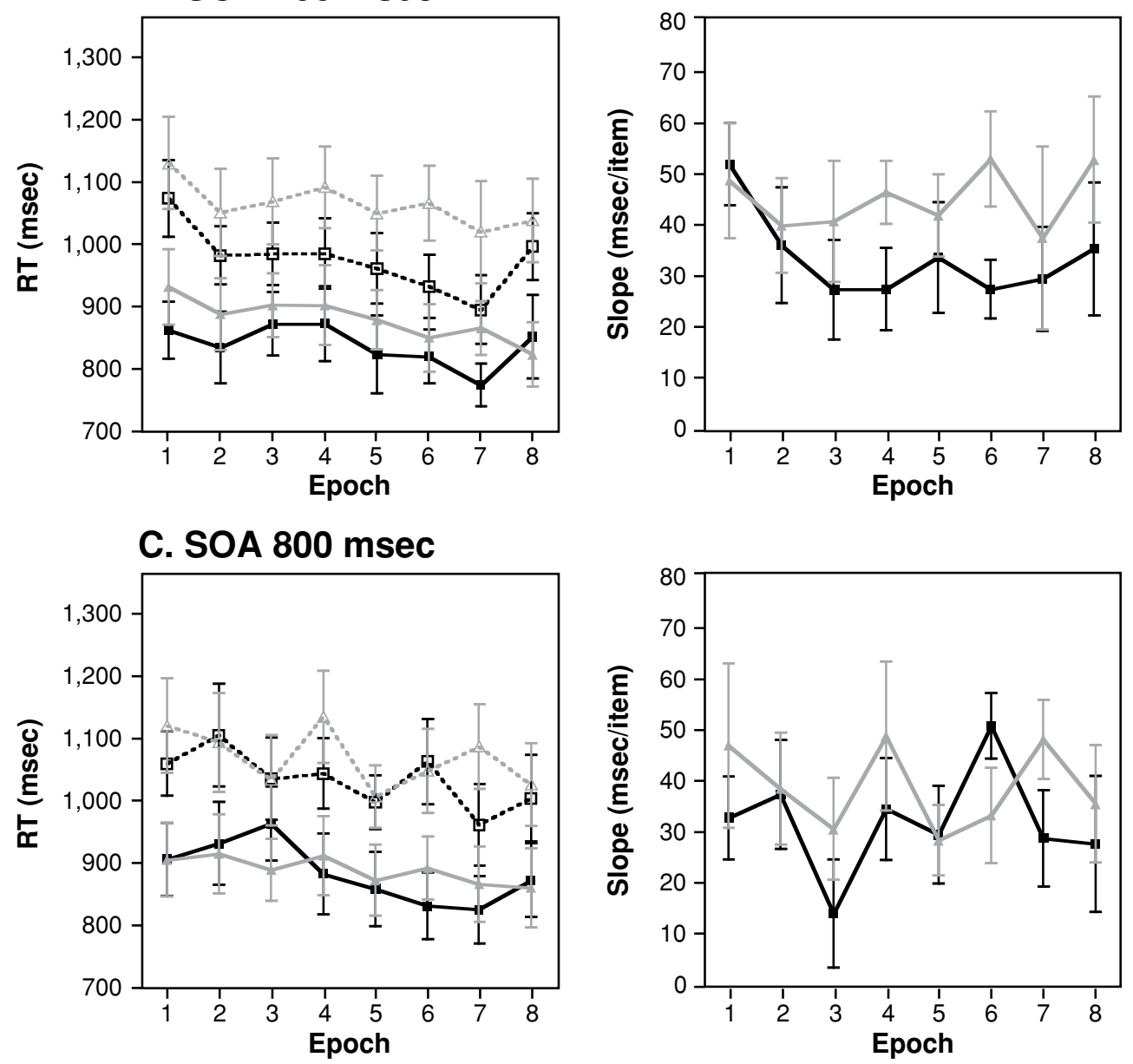

Figure 2. Mean correct reaction times (RTs) and search slopes for each SOA over epoch in Experiment 1A. 

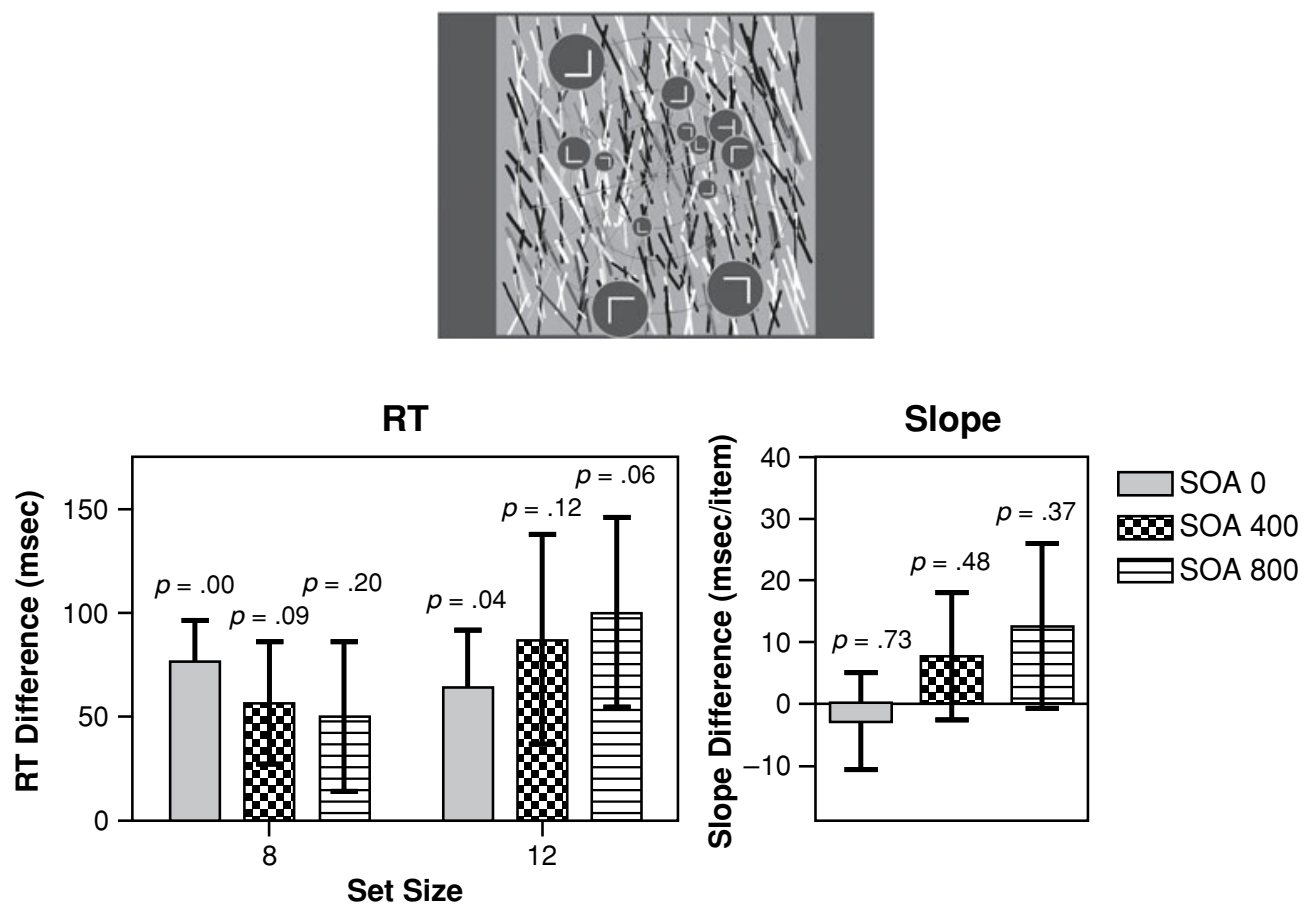

Figure 3. Example displays, reaction time (RT) differences, and slope differences for Experiment 1B. The RT differences and slope differences both reflect the difference in random (nonpredictive) and predictive trials averaged across Epochs 5-7. Here, the background texture-orientation was the predictive feature.

\section{EXPERIMENTS 1B-1F Replication and Extension}

Because the failure to find guidance was an essentially negative finding, we attempted several replications. All were similar in design to Experiment $1 \mathrm{~A}$ and are briefly described here.

\section{Method}

Experiment 1B. In this replication, the global background texture predicted the target location. The background was composed of differently orientated lines, rectangles, or squares that could be either densely or sparsely packed. All stimuli were achromatic.

Experiment 1C. In this replication, the exact color of the stimuli and their placeholders predicted target location. Participants searched for a $\mathrm{T}$ (oriented to the left or the right) among rotated Ls (similar to Experiment 1A).

Experiment 1D. In this replication, the exact color of the stimuli and their placeholders predicted target location. Participants searched for either a vertical or horizontal line among distractor lines of orientation at $-60^{\circ},-30^{\circ}, 30^{\circ}$, or $60^{\circ}$.

Experiment 1E. In this replication, the global color of the background predicted target location (as in Experiment 1A). However, the spatial layout of the display remained constant across trials (i.e., it never changed).

Experiment 1F. In this replication, the global color of the background predicted target location (as in Experiment 1A). The target was a vertical or horizontal line among oblique distractors (as in Experiment 1D). Moreover, larger set sizes were used (18 and 24).

In Experiments 1B, 1C, and 1D, the backgrounds were presented at SOAs of 0,400 , and $800 \mathrm{msec}$ prior to the onset of the search stimuli. In Experiments $1 \mathrm{E}$ and $1 \mathrm{~F}$, only SOA $0 \mathrm{msec}$ was used. Figures 3-7 give example displays of each condition. In all experiments, at least 10 participants from 18 to 55 years old took part. Each participant passed the Ishihara test for color blindness and had normal or corrected-to-normal vision. All participants gave informed consent and were paid for their time.

\section{Results and Discussion}

Figures 3-7 show the difference between predictive and nonpredictive RTs and slopes for each set size averaged across Epochs 5-7. Table 2 shows the average search slopes for predictive and nonpredictive displays, also averaged across Epochs 5-7. As we can see with SOA $0 \mathrm{msec}$, there is an RT benefit for each condition. In all cases, predictive RTs are faster than nonpredictive. A similar ef-

Table 2

Search Slopes (in Milliseconds/Item) for Experiments 1B-1F (Averaged Across Epochs 5-7)

\begin{tabular}{lccc}
\hline & & \multicolumn{2}{c}{ Condition } \\
\cline { 2 - 4 } & SOA $(\mathrm{msec})$ & Predictive & Random \\
\hline Experiment 1B & 0 & 39 & 36 \\
& 400 & 48 & 55 \\
Experiment 1C & 800 & 38 & 55 \\
& 0 & 36 & 50 \\
Experiment 1D & 400 & 34 & 70 \\
& 800 & 57 & 50 \\
Experiment 1E & 0 & 45 & 65 \\
Experiment 1F & 400 & 64 & 70 \\
& 800 & 14 & 61 \\
& 0 & 28 & 17 \\
\hline
\end{tabular}



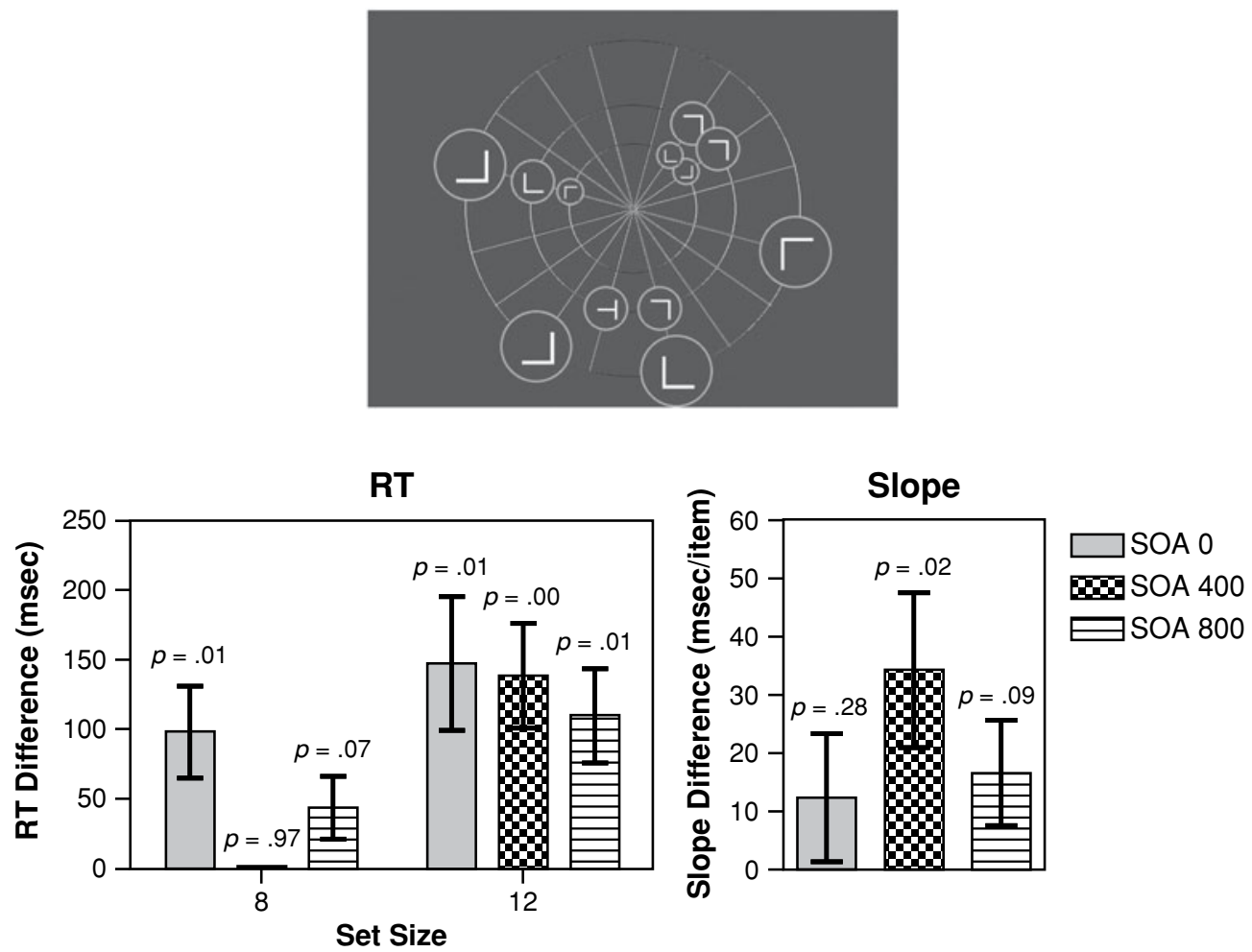

Figure 4. Example displays, reaction time (RT) differences, and slope differences for Experiment 1C. The RT differences and slope differences both reflect the difference in random (nonpredictive) and predictive trials averaged across Epochs 5-7. Here, the stimuli and placeholder color were the predictive features.

fect occurs at SOA 400 and SOA 800 msec; however, unlike that for SOA $0 \mathrm{msec}$, this result is often not reliable. This lack of statistical reliability is most pronounced for SOA 800 msec. As was demonstrated in Experiment 1A, it seems that presenting the background prior to the search stimuli might have weakened the cuing effect. We will discuss this further in the General Discussion.

Turning to the differences between predictive and nonpredictive search slopes, we see that there was - at besta weak improvement in search efficiency with predictive global features. The improvement in search efficiency was only statistically reliable when observers searched for a colored T among colored Ls in Experiment $1 \mathrm{C}$ at SOA 400 msec. $^{1}$ To investigate whether a stronger effect of guidance would be found if we increased the power, we performed a meta-analysis across experiments (using the data from Epochs 5-7). Because the SOAs and set size varied, we chose conditions that had been used in the majority of experiments (i.e., conditions that used set sizes 8 and 12 with an SOA of $0 \mathrm{msec}$ ). This allowed us to pool the data from 66 participants who had taken part in Experiments $1 \mathrm{~A}-1 \mathrm{E}$. Even with this increased power, there was still no search slope benefit of predictive context over nonpredictive $[t(65)=-1.4$, n.s.]. We also performed a similar meta-analysis on the first block of trials that each participant was tested on. This ensured that previous global-feature to target-location mappings between blocked conditions did not interfere with any potential slope effect. We pooled data across Experiments 1A-1D, where 19 participants were tested in the SOA 0 -msec condition, 18 participants in the SOA $400-\mathrm{msec}$ conditions, and 17 participants in the SOA 800 -msec conditions. The data show that even when participants had no previous exposure to the mapping of background to target location, there was no difference in search efficiency between predictive and nonpredictive backgrounds $[t(18)=-0.5$, n.s.; $t(17)=-1.7$, n.s.; and $t(16)=-1.6$, n.s., for SOAs 0,400 , and $800 \mathrm{msec}$, respectively].

Despite this lack of difference in search efficiency, 9 of the 11 individual conditions (pooled over Experiments 1B-1F) produced (nonreliable) changes in the direction of improvement. In the most dramatic case, Experiment $1 \mathrm{~F}$, there was an impressive $50-\mathrm{msec} /$ item difference in slope between predictive and nonpredictive search slopes. Although it was only marginally significant ( $p=.06$ ), the bulk of participants seemed to show an effect. As is shown in Figure 8, the large size was driven by 2 participants who both showed a 150 - to $170-\mathrm{msec} /$ item difference. The lack of significance was driven by 2 other participants whose results went in the opposite direction. It may be noteworthy that Experiment $1 \mathrm{~F}$ was the hardest search task of the group: a consequence of larger set sizes and of the similarity between targets and distractors (Duncan \& Humphreys, 1989). As a result, RTs were quite slow (averaging 2,230 msec across conditions). This might have given participants enough time to finally be able to use the global feature information for guidance. 

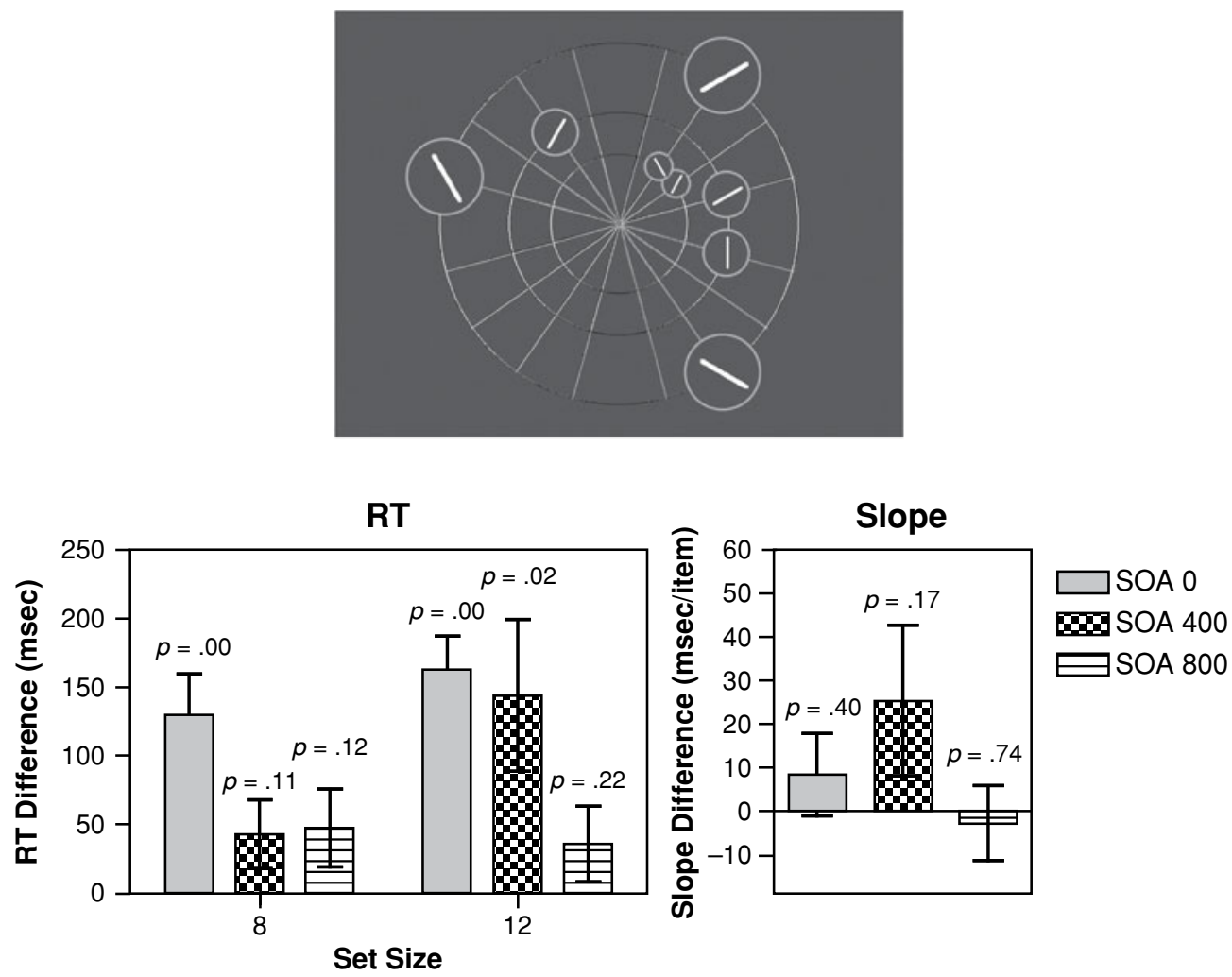

Figure 5. Example displays, reaction time (RT) differences, and slope differences for Experiment 1D. The RT differences and slope differences both reflect the difference in random (nonpredictive) and predictive trials averaged across Epochs 5-7. Here, the stimuli and placeholder color were the predictive features. The target in this condition was either a horizontal or vertical line, and the distractors were made up of oblique lines oriented at $-60^{\circ},-30^{\circ}, 30^{\circ}$, or $60^{\circ}$.

This idea was confirmed in Experiment 2, where guidance by global features was possible if participants were given enough time and explicit instruction.

In summary, Experiment 1-in all of its forms-revealed that global feature information can speed mean RT. Its ability to guide search and thereby increase search efficiency, however, appears to be more limited.

\section{EXPERIMENT 2}

\section{Does Explicit Knowledge Aid Guidance?}

Is there something fundamentally wrong with the idea that the attributes of the background could inform the observer about the probable location of that target? In Experiment 2 , we investigated whether featural properties of the display as a whole could ever guide the deployment of attention. In this experiment, we gave participants explicit knowledge that a background was predictive (e.g., telling them that if the overall background is red, a target would appear in location X). Moreover, we increased the SOA between background and search display to $1,500 \mathrm{msec}$ in order to give participants adequate preparation time to orient themselves to the expected target location. Given this information, it seemed unlikely that participants would still feel the need to search for the target. Therefore, we expected search slopes for predictive displays to be shallower than those for random displays (i.e., a guidance effect). This experiment was supposed to show us what the results of Experiment 1 would have looked like if overall image properties had guided attention.

\section{Method}

Participants. Fourteen participants from 18 to 55 years old served as participants. Each participant passed the Ishihara test for color blindness and had normal or corrected-to-normal vision. All participants gave informed consent and were paid for their time.

Stimuli and Procedure. The stimuli and procedure were the same as in Experiment 1A. However, participants were tested with only a single SOA of $1,500 \mathrm{msec}$ between the onset of the background and the onset of the search stimuli. Furthermore, participants were given explicit instructions informing them that four of the background colors were predictive of the target location, whereas the other four colors were not. They were told exactly which four colors would be predictive (e.g., red, purple, green, and orange). As in Experiment 1A, the four colors predicted the target locations in Epochs 1-7. In Epoch 8, the target locations in all conditions were randomized so that the predictive background colors no longer predicted the target location.

\section{Results and Discussion}

Overall error rates were low $(2 \%)$ and are presented in Table 1. Because they were not indicative of any speed- 

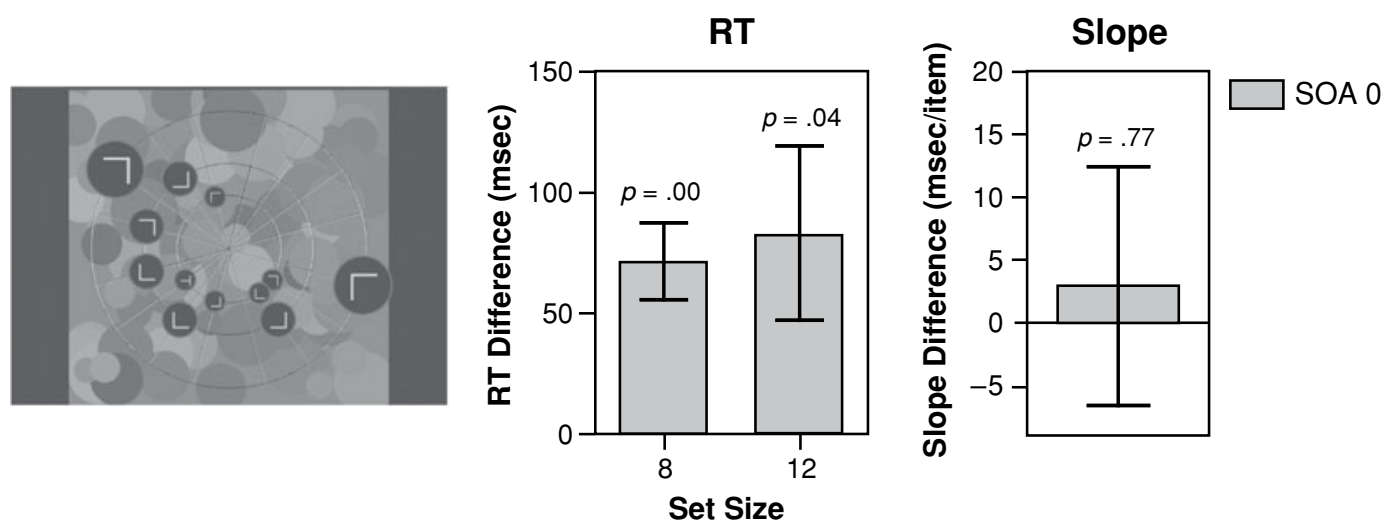

Figure 6. Example displays, reaction time (RT) differences, and slope differences for Experiment 1E. The RT differences and slope differences both reflect the difference in random (nonpredictive) and predictive trials averaged across Epochs 5-7. Here, the background color was the predictive feature. The spatial configuration of the display remained constant throughout the experiment.

accuracy trade-off, we will not discuss them further and will instead concentrate on RT and slope analysis. RTs below $200 \mathrm{msec}$ and above $4,000 \mathrm{msec}$ were eliminated. This led to the removal of less than $1 \%$ of the data. Figure 9 shows the RT and slope data, respectively, for both predictive and random configurations. There was a main effect of having a predictive background. This occurred for both set size $8[F(1,13)=37.6, p<.01]$ and set size $12[F(1,13)=26.4, p<.01]$. In comparing predictive and random trials for RTs collapsed across Epochs 5-7, we see that there was a feature cuing effect of $324 \mathrm{msec}$ for set size $8[t(13)=-5.8, p<.01]$ and $459 \mathrm{msec}$ for set size $12[t(13)=-5.2, p<.01]$. There was no difference between predictive and random RTs in Epoch 8 (for both set sizes 8 and 12), where none of the backgrounds remained predictive. Clearly, as in Experiment 1A, participants were faster when the background color predicted the target location as opposed to when it did not.

However, unlike in Experiment 1A, there was also a positive guidance effect. There was a strong main effect of search slope $[F(1,13)=10.2, p<.01]$, and averaging slopes across Epochs 5-7 showed a 34-msec/item advantage in search efficiency with predictive backgrounds as opposed to random backgrounds [ $t(13)=-2.8, p<.05]$. In this situation, prior knowledge helped guidance. As mentioned briefly in the introduction, an increase in guidance is one attentional model that can account for the reduction in predictive slopes. This is not to say that the data cannot be accounted for by other attention models (such as limitedresource models of attention - see, e.g., Posner, 1980; Posner \& Petersen, 1990; or unlimited-capacity models - see, e.g., Palmer, 1994; Palmer et al., 1993; Palmer et al., 2000; Shaw \& Shaw, 1977; Townsend \& Ashby, 1983). Regardless of which attention model is accountable, however, the important point remains that search slopes can be dramatically reduced when participants are given time and explicit knowledge about predictive contexts.

Also noteworthy is the fact that search slopes for predictive trials did not reach 0 msec/item. Rather, they became asymptotic at around $20 \mathrm{msec} /$ item. Breaking down
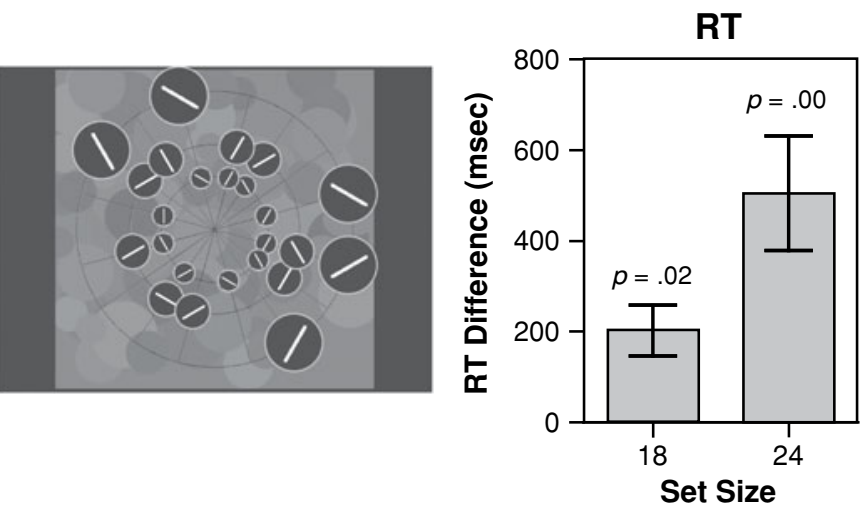

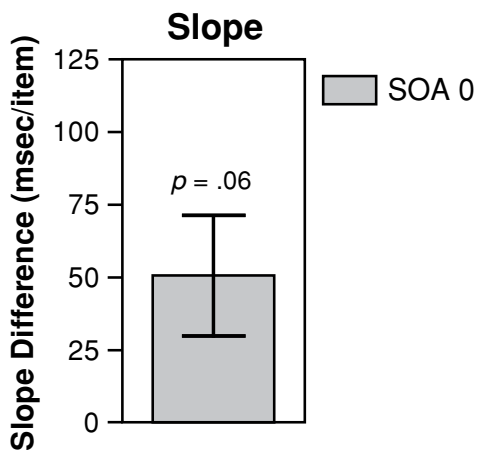

Figure 7. Example displays, reaction time (RT) differences, and slope differences for Experiment 1F. The RT differences and slope differences both reflect the difference in random (nonpredictive) and predictive trials averaged across Epochs 5-7. Here, the background color was the predictive feature. The target in this condition was either a horizontal or vertical line, and the distractors were made up of oblique lines oriented at $-6^{\circ},-30^{\circ}, 30^{\circ}$, or $60^{\circ}$. The set size on a giving trial was either 18 or 24 items. 


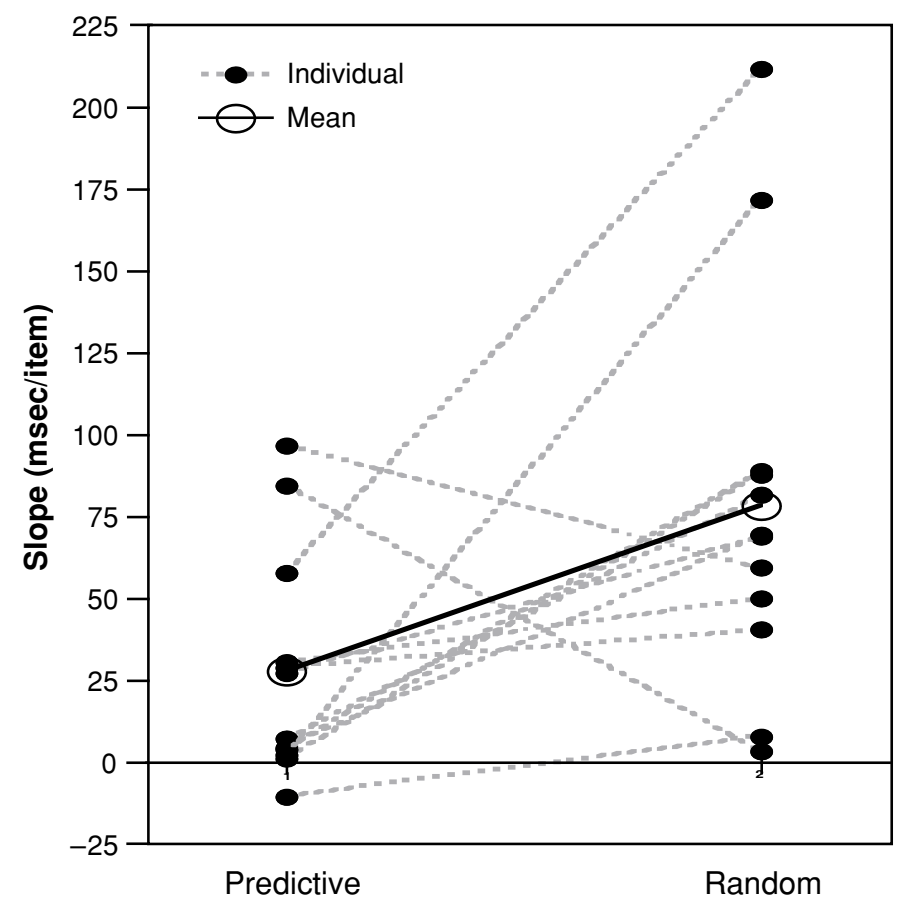

Figure 8. Individual search slopes for each participant in Experiment $1 \mathrm{~F}$ for both predictive and nonpredictive (random) trials.

the data and looking at individual participants, we see that $64 \%$ of participants reached $0 \mathrm{msec} /$ item in slope efficiency by the latter epochs, whereas the other $36 \%$ did not. Similar effects have been shown in the contextual cuing literature, which has reported that approximately $30 \%$ of participants do not show a standard contextual cuing effect (see, e.g., Jiang, Leung, \& Burks, 2006; Lleras \& von Mühlenen, 2004). In contrast, just $20 \%$ of participants overall had slopes near zero in Experiments $1 \mathrm{~A}-1 \mathrm{~F}$ ( $0 \%$ in both Experiments $1 \mathrm{~A}$ and $1 \mathrm{~B} ; 13 \%$ in both Experiments $1 \mathrm{C}$ and $1 \mathrm{D} ; 43 \%$ in Experiment $1 \mathrm{E}$; and $50 \%$ in Experiment 1F. Presumably, the relatively high number of participants producing efficient slopes in Experiment $1 \mathrm{~F}$ reflects the notion that guidance by global features is relatively slow and may appear when participants are given lots of time, as well as explicit knowledge).

\section{GENERAL DISCUSSION}

During visual search, a predictive spatial layout can be used to speed target response (see, e.g., Chun \& Jiang, 1998, 2003). We conducted several experiments to investigate whether global featural properties of the display-in the absence of a consistent spatial layout - can be used to (1) cue the target location and thus speed RT response and (2) guide attention to the target item, resulting in shallower search slopes. In Experiment 1A, we investigated whether the average background color could cue and/or guide search. In this case, the background was presented either concurrently with or in advance of (e.g., $400 \mathrm{msec}$ or $800 \mathrm{msec}$ prior) the stimulus onset. The results showed that when the background was presented simultaneously with the stimuli (SOA $0 \mathrm{msec}$ ), RTs were faster with predictive background features than with nonpredictive background features. A similar effect occurred when the background appeared prior to the stimuli: RTs were faster when the background was predictive rather than random. This effect remained reliable when the background appeared $400 \mathrm{msec}$ prior to the onset of the search items. When the SOA increased to $800 \mathrm{msec}$, the effect was less reliable (only marginally significant at set size 8). Experiments $1 \mathrm{~B}-1 \mathrm{~F}$ showed a similar pattern of results. In these experiments, we varied the nature of the predictive feature to include background texture-orientation and stimulus color. We also varied the nature of the search task and the set size. The basic pattern of the data, however, remained unchanged. Collectively, the results of Experiment 1 show that a predictive display speeds RT.

In comparison with the evidence for a main effect of background on RT, the evidence for guidance in Experiments $1 \mathrm{~A}-1 \mathrm{~F}$ was much less convincing. If one looks at the whole set of data, one may get the impression that there is some guidance of attention. The slopes of RT $\times$ set size functions seem more likely to be shallower in the predictive cases than in the nonpredictive cases. However, this effect - if it is real - is statistically fragile. The important point is that guidance by simple feature backgrounds is nothing like guidance by simple target features. Imagine an experiment in which observers search for a $\mathrm{T}$ of arbitrary color among Ls of other arbitrary colors. With stimuli like those used in Experiment 1A, the RT $\times$ set size slope would be about $30-40 \mathrm{msec} /$ item. If, however, 

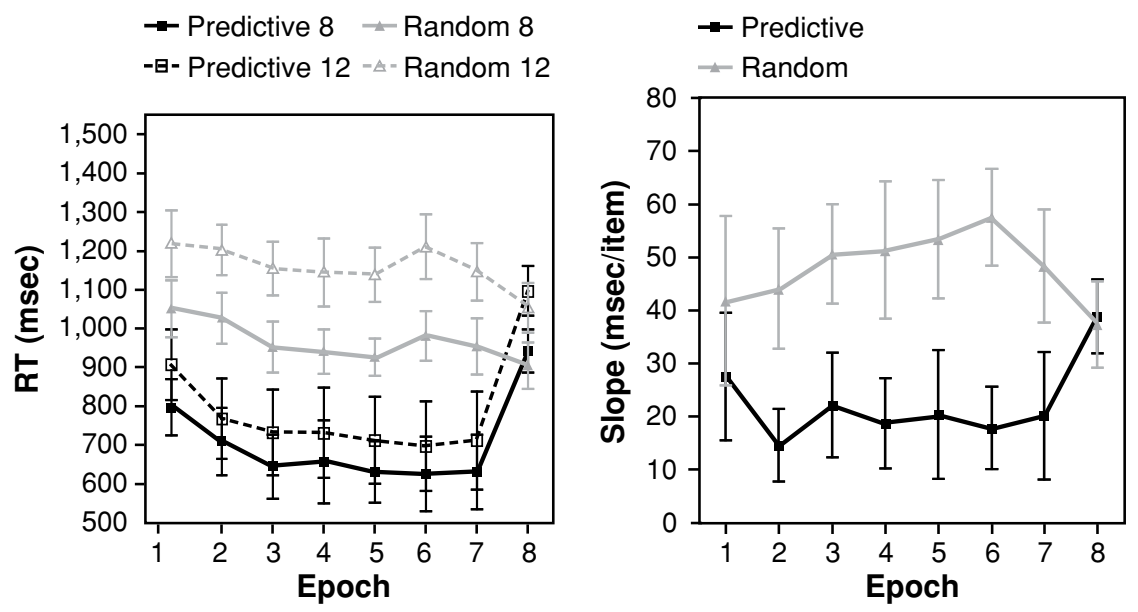

Figure 9. Mean correct reaction times (RTs) and search slopes (milliseconds/item) over epoch for Experiment 2; SOA was 1,500 msec. Participants were told in advance which background colors were predictive.

the target $\mathrm{T}$ is always red, the slope would drop dramatically. With the appropriate choice of distractor colors, the slope would drop to near zero. That is perfect guidance by target color. Suppose instead that the target, T, is always in a specific location when the display (as a whole) is red. In both cases, red would tell the observer where to look for the target, but the results of the present experiment show that the global redness would provide little - if any-guidance, unless the participant is given sufficient time and explicitly told to use the information.

It is possible that the predictive global feature assignments in these experiments were too weak. Half the global features were predictive of the target location, whereas the other half were not. If participants were learning that the global feature was valid only $50 \%$ of the time, they might also have learned that it was not a reliable cue and chosen not to use it. Although this is possible, we do not believe this to be the case. In a separate experiment, we investigated what would happen to the search slope if all the displays were predictive. Even in this case, search slopes did not become more efficient over time $[F(6,72)=0.9$, n.s. $]$. Please note also that an RT benefit occurred in all the experiments reported above, showing that repeating the nonpredictive feature does not disrupt RT contextual learning. This replicates most of the findings in the contextual cuing literature, which concentrate on RTs rather than on search slopes (see Chun \& Jiang, 1998, for the exception). Interestingly, in other work, we have also looked for the effect on search slopes of standard contextual cuing by spatial layout. The results were similar to those presented here. We replicated the standard reduction in mean RT, but there is little effect of predictive configurations on search slopes (Kunar, Flusberg, Horowitz, \& Wolfe, in press).

Another suggestion explaining the lack of guidance is that in the present studies, participants were not actively attending the predictive global features. Previous research has found that unattended stimuli do not produce contextual cuing (Jiang \& Chun, 2001; Jiang \& Leung, 2005).
This may explain why a strong guidance effect occurred only in Experiment 2, when participants were given explicit knowledge and encouraged to attend the backgrounds. Although this hypothesis has some merits, it cannot account for all of the data. In the experiments reported here, although there was little benefit to guidance when the background features were not explicitly attended, there was an RT effect. This suggests that the global features were being attended, at least to some degree: In the previous studies, where context was unattended, no RT benefit was found (Jiang \& Chun, 2001; Jiang \& Leung, 2005). Of course, it could be the case that the feature mismatch in these studies was the reason for little guidance benefit. In the standard contextual cuing studies, the spatial configuration of the display is said to guide attention to the spatial target location. However, in the studies presented here, the guiding feature was nonspatial. This raises an interesting point: Perhaps guidance to spatial target locations can be found only with a predictive spatial cue? Although this is possible, other studies in our lab have suggested otherwise. As we mentioned briefly above, even when the predictive cue was spatial, we found that there was little benefit to guidance (Kunar et al., in press).

It is curious that increasing the timing between the predictive feature onset and the stimulus onset seemed to weaken the RT cuing effect. A positive cuing effect was always found in the SOA 0 -msec conditions but not always in the SOA 400- or 800-msec conditions. ${ }^{2}$ At present, we are not sure why this should be. One possibility could be our use of different layouts in the backgrounds. For example, in Experiment 1A, the global feature of a red background would have predicted the target appearing in location X. However, no two red backgrounds were identical (i.e., each background was composed of randomly generated luminance values that produced the overall impression of red but were individually unique). With an SOA of $0 \mathrm{msec}$, perhaps participants had time only to analyze the global properties of the background (i.e., an overall im- 
pression of red) leading to a strong RT effect. With longer SOAs, participants may have had time to process the local features within each display as well. As these local features changed from trial to trial, their inconsistency could have interfered with the global contextual cuing effect. Although this may explain some of the data, it cannot account for the results of Experiments 1C and 1D. A weakened contextual cuing effect occurred with longer SOAs in these conditions; however, the specific local features around the target (i.e., the color of the stimuli and their placeholders for each predictive feature) did not change. A second possibility suggests that the association between predictive context and target location is strongest when they temporally group. Therefore, the strongest cuing effect would occur at SOA $0 \mathrm{msec}$, whereas it might dissipate somewhat at SOA $400 \mathrm{msec}$ and $800 \mathrm{msec}$. However, further research needs to be conducted before any firm conclusions can be reached.

The data presented here show that global featural properties (in the absence of explicit knowledge) speed RT, but do not guide search effectively. If the RT-speeding advantage is not due to guidance, then what is facilitating RTs? Perhaps the RT benefit is due to a reduction in target processing time. Given the predictive context, it may be that less evidence needs to be accumulated before a correct response can be made. Studies in other fields concur with this hypothesis. For example, when looking at eye movements in scene recognition, Hidalgo-Sotelo, Oliva, and Torralba (2005) found that when a scene predicted the target location, the gaze duration (i.e., the amount of time a participant gazed at the target before responding) was reduced over time. ${ }^{3}$ This suggests that participants required less evidence about the target before they would commit to a correct response. Likewise, pilot data in our lab has shown that when there is no target in a predictive context (i.e., a target absent trial), there is no contextual cuing. In this case, since there is no target, its recognition cannot be facilitated. Future work needs to investigate this further.

Our present results can also give us insight as to what we mean by context. In their initial paper, Chun and Jiang (1998) found that a given distractor configuration could cue the target location: The "classical" contextual cuing effect. However, since then, other research has suggested that different aspects of the scene context can also speed target response. For example, Song and Jiang (2005) have found that only the invariant context of approximately three of these distractors is needed to produce a contextual cuing effect. Furthermore, the configuration of two individual displays can be recombined to produce a "novel" context and still produce valid cuing (Jiang \& Wagner, 2004). Work from our lab has shown that the overall context need not involve distractor items; rather, it can be marked out by placeholders (Kunar, Michod, \& Wolfe, 2005). Interactions between the spatial layout of the distractors and background type also accrue. DiMase and Chun (2004) found that when the spatial layout of the display was invariant across all trials, a predictive scene in the background could produce effective cuing of target position, whereas Neider and Zelinsky (2005) found that tar- gets were found faster if a scene semantically constrained the target location. Similarly, Hyun and Kim (2002) found that both the predictive spatial layout and its background were implicitly learned and useful in contextual cuing (see also Yokosawa \& Takeda, 2004, who found that combining a consistent background with a spatial layout produces stronger contextual cuing). The present results take this one step further and say that, in the absence of useful spatial information, predictive global featural aspects of the display (whether the background or stimuli properties) can also produce a valid contextual cuing effect. It seems that global features can serve as the context in contextual cuing. They only serve as guiding features, however, when their association with target location is made explicit and when enough time is provided.

\section{REFERENCES}

Biederman, I. (1972). Perceiving real-world scenes. Science, 177, 77-80. Biederman, I., Glass, A. L., \& Stacey, E. W., Jr. (1973). Searching for objects in real-world scenes. Journal of Experimental Psychology, 97, 22-27.

BrainaRd, D. H. (1997). The Psychophysics Toolbox. Spatial Vision, 10, 433-436.

BraUn, J., \& SAGI, D. (1990). Vision outside the focus of attention. Perception \& Psychophysics, 48, 45-58.

CARTER, R. C. (1982). Visual search with color. Journal of Experimental Psychology: Human Perception \& Performance, 8, 127-136.

Chun, M. M. (2000). Contextual cueing of visual attention. Trends in Cognitive Sciences, 4, 170-178.

Chun, M. M., \& Jiang, Y. (1998). Contextual cueing: Implicit learning and memory of visual context guides spatial attention. Cognitive Psychology, 36, 28-71.

ChUn, M. M., \& JiAng, Y. (2003). Implicit, long-term spatial contextual memory. Journal of Experimental Psychology: Learning, Memory, \& Cognition, 29, 224-234.

DiMase, J. S., \& Chun, M. M. (2004). Contextual cueing by real-world scenes [Abstract]. Journal of Vision, 4, 259a.

Duncan, J., \& Humphreys, G. W. (1989). Visual search and stimulus similarity. Psychological Review, 96, 433-458.

Egeth, H., \& Dagenbach, D. (1991). Parallel versus serial processing in visual search: Further evidence from subadditive effects of visual quality. Journal of Experimental Psychology: Human Perception \& Performance, 17, 551-560.

GreEn, B. F., \& ANDERSON, L. K. (1956). Color coding in a visual search task. Journal of Experimental Psychology, 51, 19-24.

Hidalgo-Sotelo, B., Oliva, A., \& Torralba, A. (2005). Human learning of contextual priors for object search: Where does the time go? In Proceedings of the 3rd Workshop on Attention and Performance. San Diego: International Conference on Computer Vision and Pattern Recognition.

Hyun, J., \& Kim, M. (2002). Implicit learning of background context in visual search. Technical Report on Attention \& Cognition, 7, 1-4.

JiAng, Y., \& ChUN, M. M. (2001). Selective attention modulates implicit learning. Quarterly Journal of Experimental Psychology, 54A, 1105-1124.

JiANG, Y., \& LEUNG, A. W. (2005). Implicit learning of ignored visual context. Psychonomic Bulletin \& Review, 12, 100-106.

Jiang, Y., Leung, A. [W.], \& Burks, S. (2006). Source of individual differences in spatial context learning. Manuscript submitted for publication.

JIANG, Y., \& WAGNER, L. C. (2004). What is learned in spatial contextual cuing - configuration or individual locations? Perception \& Psychophysics, 66, 454-463.

Kunar, M. A., Flusberg, S. J., Horowitz, T. S., \& Wolfe, J. M. (in press). Does contextual cueing guide the deployment of attention? Journal of Vision.

Kunar, M. A., Michod, K. O., \& Wolfe, J. M. (2005). When we use the context in contextual cueing: Evidence from multiple target locations [Abstract]. Journal of Vision, 5, 412a. 
Lleras, A., \& VON MÜHLEnen, A. (2004). Spatial context and top-down strategies in visual search. Spatial Vision, 17, 465-482.

NeIDER, M. B., \& ZELINSKY, G. J. (2005). Effects of scene-based contextual guidance on search [Abstract]. Journal of Vision, 5, 414a.

PAlmer, J. (1994). Set-size effects in visual search: The effect of attention is independent of the stimulus for simple tasks. Vision Research, 34, 1703-1721.

Palmer, J., Ames, C. T., \& Lindsey, D. T. (1993). Measuring the effect of attention on simple visual search. Journal of Experimental Psychology: Human Perception \& Performance, 19, 108-130.

Palmer, J., Verghese, P., \& Pavel, M. (2000). The psychophysics of visual search. Vision Research, 40, 1227-1268.

Pelli, D. G. (1997). The VideoToolbox software for visual psychophysics: Transforming numbers into movies. Spatial Vision, 10, 437-442.

Posner, M. I. (1980). Orienting of attention. Quarterly Journal of Experimental Psychology, 32, 3-25.

Posner, M. I., \& Petersen, S. E. (1990). The attention system of the human brain. Annual Review of Neuroscience, 13, 25-42.

Shaw, M. L., \& Shaw, P. (1977). Optimal allocation of cognitive resources to spatial locations. Journal of Experimental Psychology: Human Perception \& Performance, 3, 201-211.

Song, J.-H., \& JiANG, Y. (2005). Connecting the past with the present: How do humans match an incoming visual display with visual memory? Journal of Vision, 5, 322-330.

Townsend, J. T., \& Ashby, F. G. (1983). The stochastic modeling of elementary psychological processes. New York: Cambridge University Press.

Treisman, A., \& Gelade, G. (1980). A feature-integration theory of attention. Cognitive Psychology, 12, 97-136.

Treisman, A., \& Sato, S. (1990). Conjunction search revisited. Journal of Experimental Psychology: Human Perception \& Performance, 16, 459-478.

Wolfe, J. M. (1994). Guided Search 2.0: A revised model of visual search. Psychonomic Bulletin \& Review, 1, 202-238.

Wolfe, J. M. (1998). What can 1 million trials tell us about visual search? Psychological Science, 9, 33-39.

Wolfe, J. M., Cave, K. R., \& Franzel, S. L. (1989). Guided Search: An alternative to the feature integration model for visual search. Journal of Experimental Psychology: Human Perception \& Performance, 15, 419-433.

Wolfe, J. M., \& Horowitz, T. S. (2004). What attributes guide the deployment of visual attention and how do they do it? Nature Reviews Neuroscience, 5, 495-501.

Wolfe, J. M., Oliva, A., Horowitz, T. S., Butcher, S. J., \& Bompas, A. (2002). Segmentation of objects from backgrounds in visual search tasks. Vision Research, 42, 2985-3004.

Yokosawa, K., \& TAKEDA, Y. (2004). Combination of background and spatial layout produces a stronger contextual cueing effect [Abstract]. Journal of Vision, 4, 686a.

\section{NOTES}

1. A similar pattern occurs if we collapse the data across Epochs 3-7. Here, out of the fourteen conditions from Experiments $1 \mathrm{~A}-1 \mathrm{~F}$, ten of the conditions showed that there was no benefit in search slope between predictive and random trials. One showed that there was a marginal slope benefit of having a predictive context over a random context (Experiment 1B-SOA $800 \mathrm{msec}, p=.09$ ), whereas the other three conditions showed a reliable slope benefit (Experiment 1A-SOA $400 \mathrm{msec}$, Experiment 1C-SOA $400 \mathrm{msec}$, and Experiment 1F). Again, the data confirmed that any improvement in search efficiency was - at best-weak, and that the majority of experiments showed no benefit at all.

2. These data are puzzling and perhaps suggest that the explicit instruction in Experiment 2 was more important than the increased SOA for producing a guidance benefit. Although this might be the case, we do not want to rule out the effect of SOA entirely. Please remember that a marginal guidance benefit was also found in Experiment 1F, where - due to increased response times - there was also more time to encode the background.

3. Since this study investigated search within a scene, there was no direct measure of set size. Further research would need to be conducted before we could speculate on set size $\times$ RT interactions (i.e., an interaction could occur if gaze durations for distractor items also decreased across repeated displays).

(Manuscript received June 2, 2005; revision accepted for publication December 13, 2005.) 